\title{
Acrosome reaction in the cumulus oophorus revisited: involvement of a novel sperm-released factor NYD-SP8
}

\author{
Ting Ting Sun, Chin Man Chung, Hsiao Chang Chan ${ }^{凶}$ \\ Epithelial Cell Biology Research Center, School of Biomedical Sciences, Faculty of Medicine, The Chinese University of Hong \\ Kong, Hong Kong, China \\ 凶 Correspondence: hsiaocchan@cuhk.edu.hk \\ Received January 31, 2011 Accepted February 7, 2011
}

\begin{abstract}
Fertilization is a process involving multiple steps that lead to the final fusion of one sperm and the oocyte to form the zygote. One of the steps, acrosome reaction (AR), is an exocytosis process, during which the outer acrosome membrane fuses with the inner sperm membrane, leading to the release of acrosome enzymes that facilitate sperm penetration of the egg investments. Though AR has been investigated for decades, the initial steps of $A R$ in vivo, however, remain largely unknown. A well elucidated model holds the view that AR occurs on the surface of the zona pellucida (ZP), which is triggered by binding of sperm with one of the ZP glycosylated protein, ZP3. However, this model fails to explain the large number of 'falsely' acrosome-reacted sperms found within the cumulus layer in many species examined. With the emerging evidence of cross-talk between sperm and cumulus cells, the potential significance of $A R$ in the cumulus oophorus, the outer layer of the egg, has been gradually revealed. Here we review the acrosome status within the cumulus layer, the cross-talk between sperm and cumulus cells with the involvement of a novel spermreleased factor, NYD-SP8, and re-evaluate the importance and physiological significance of the $A R$ in the cumulus in fertilization.
\end{abstract}

KEYWORDS sperm, acrosome reaction, cumulus, NYP-SP8, progesterone

\section{INTRODUCTION}

Fertilization is a multi-step process that leads to eventual interaction of sperm and oocyte and formation of a zygote. To reach and fertilize an oocyte, millions of spermatozoa travel a long way, through the cervix to the oviduct, in the female reproductive tract, and undergo a series of critical events including capacitation, acrosome reaction (AR), penetration through the oocyte vestments, until one out of the millions of spermatozoa fuses with and enters the oocyte to form the zygote.

The mature oocyte is surrounded by two investments. The outer layer is the cumulus oophorus composed of cumulus cells and matrix, and the inner layer is known as the zona pellucida (ZP) outside of the egg membrane. It is generally accepted that in order to fertilize the egg, spermatozoa have to go through the following sequence of events to penetrate through egg investments: (1) sperm capacitation in the fallopian tube/oviduct; (2) sperm penetration through the cumulus; (3) binding of acrosome-intact sperm to ZP; (4) AR; (5) penetrating ZP by acrosome-reacted sperm; and (6) fusion with the egg (Yanagimachi, 1994).

Although sperm morphology differs in species, all spermatozoa contain a single Golgi-derived secretory granule located at their anterior head, called the acrosome. AR, one of the critical events in mammal fertilization, is defined as the fusion of the outer acrosome membrane with inner spermatozoa plasma membrane in multiple sites (Yanagimachi, 1994; Brucker and Lipford, 1995; Ikawa et al., 2010). In response to appropriate stimuli, the outer acrosome membrane and the overlying sperm plasma membrane fuse and vesiculate, leading to exposure of acrosomal contents. During this exocytosis process, the hydrolytic enzymes such as hyaluronidase and acrosin within acrosome are released from spermatozoa as a result of AR (Tulsiani et al., 1998).

The mechanisms underlying AR have been extensively investigated, which are believed to be mediated mainly via extracellular calcium influx (Fukami et al., 2001; Breitbart 
et al., 2010); however, the initial steps of AR in vivo remain largely unclear. Most researchers agree that AR occurs on the surface of ZP layer of the oocyte when a capacitated sperm encounters $\mathrm{ZP}$, and is triggered by one of the glycosylated protein, ZP3 (Cross et al., 1988; Lee et al., 1992; van Duin et al., 1994; Gupta et al., 2009). This model assumes that the acrosome is either intact or reacted when spermatozoa reach ZP, and only those with intact acrosome can bind with ZP3 and carry out AR. The hydrolytic enzymes released from the acrosome help the sperm penetrate the ZP layer and reach the egg. However this model cannot explain why quite a number of spermatozoa in the cumulus oophorus are already acrosome-reacted, before they get to ZP (Bedford, 1972; Cummins and Yanagimachi, 1982). Recently, accumulating evidence of cross-talk between the cumulus oophorus and spermatozoa has emerged (Yin et al., 2009; Tanii et al., 2011), suggesting a more important role of the cumulus matrix and cumulus cells in fertilization, which has been long overlooked. Herein we re-examine evidences related to $A R$ in the cumulus oophorus, taking into account of the recent discovery of a sperm factor, NYD-SP8, released upon sperm-cumulus interaction, and discuss the molecular basis underlying the interaction of sperm and the cumulus and its potential physiological significance in fertilization.

\section{STATUS OF SPERM ACROSOME IN THE CUMULUS: THE CONTROVERSY}

To find out where AR is initiated in vivo, various methods have been adopted to observe the morphology and the ultrastructure of acrosome at the head of spermatozoa located in the ampulla of fallopian tube, the cumulus mass and the ZP layer (Pereda and Coppo, 1985; Nakanishi et al., 1999; Hasuwa et al., 2010). In spite of the differences in animal species examined and methodologies adopted, it is consistently found that the spermatozoa in the oviduct are acrosome-intact or occasionally reacted, while the spermatozoa penetrating the zona are acrosome-reacted. The experimental results vary, however, as regard to the acrosome status of spermatozoa within the cumulus or at the surface of the zona. Earlier electron microscope studies of in vivo fertilization in the rabbit have found that a majority of sperm passing between the cumulus cells displayed various stages of $A R$, while only occasionally intact sperms were present close to the ZP. Besides, the contents of the acrosome were lost before the sperm penetrated the ZP (Bedford, 1968). Similar to the rabbit, human spermatozoa found in the cumulus cells were all acrosome reacted under electron microscope with no evidence of the presence of spermatozoa with intact acrosome in the cumulus matrix (Pereda and Coppo, 1985). On the contrary, electron microscope studies of spermatozoa recovered from sheep and cow intracervical insemination showed an opposite result. Remnants or vesiculated products of AR were present at the ZP surface of every penetrated egg, which indicated that the $A R$ in sheep and cow occurs at the surface of the ZP (Crozet and Dumont, 1984a; Crozet, 1984b). This is also consistent with in vitro studies in the hamster, which suggested that fertilization in the hamster is accomplished by a spermatozoon that undergoes an AR at the surface of the ZP (Gwatkin et al., 1976). It is important to note that at the time of recovery most of these ovulated oocytes were corona cell free. The exclusion of the spermatozoa within the cumulus from these studies might cause a bias when analyzing the sites of AR in vivo, since the cumulus cells are still present around the egg at the point of fertilization in most species. Interestingly, using transgenic mice that express GFP protein in sperm acrosome, it has been feasible to observe the real-time process of AR in live sperm excluding any exoteric treatment (Nakanishi et al., 1999; Nakanishi et al., 2001; Hasuwa et al., 2010), which shed new light on finding out initial site of $A R$ in vivo.

Although the exact site of AR could not be concluded from the above mentioned conflicting in vivo and in vitro results, it does suggest that the cumulus and zona are the two most possible candidate sites for AR. Correspondently, there are two models of $A R$ with regard to its initiation and sperm penetration sites. The early model was that AR occurs in the cumulus layer before sperm penetrate the zona layer. It is the hydrolytic enzymes released following the AR that ensure sperms to disperse the cumulus mass and reach the zona layer (Talbot, 1985; Yanagimachi, 1988). Unfortunately, this hypothesis is challenged by the observation that the expression of hydrolytic enzymes thought to facilitate sperm penetration of the cumulus, $\mathrm{PH}-20$ and Hyal5, were found on the surface of sperm membrane but not within the acrosome (Lin et al., 1994; Kim et al., 2005; Kimura et al., 2009), which means sperm do not necessarily undergo AR to release hydrolytic enzymes when passing through the cumulus layer. Recent results obtained from $\mathrm{PH}-20$ null mice have revealed that mouse sperm lacking $\mathrm{PH}-20$ are still fertile with only delayed penetration through the cumulus (Baba et al., 2002), while sperm lacking both $\mathrm{PH}-20$ and Hyal5 have not yet been studied. It is possible that other hydrolytic enzymes on the sperm membrane might be involved in passing the cumulus, or these membrane enzymes are not sufficient for sperm penetration of the cumulus. Nevertheless, this AR model still lacks the key evidence for the underlying mechanisms, including the molecules triggering AR within the cumulus and mediating the interaction between sperms and the cumulus mass.

A new AR model, which holds the opinion that $A R$ is initiated at the surface of the $\mathrm{ZP}$, has gradually gained acceptance with the identification of a responsible protein involved (Yanagimachi, 1994). A glycoprotein of the ZP, ZP3, has been proved to induce the reactionary events when it binds to the membrane receptor of acrosome-intact sperm (Bleil and Wassarman, 1980; Bleil and Wassarman, 1983). 
One of the most persuasive proofs is that only acrosomeintact spermatozoa can bind to and penetrate ZP for further fusion with the inner oocyte (Miller et al., 1992), suggesting that AR must occur after binding to ZP. Thus, this widely accepted model considers the observed acrosome-reacted sperm traversing the cumulus as "false-reacted" or "spontaneous reacted" which might stick to the cumulus mass and eventually be phagocytosed by the cumulus cells. However, a recent capillary-cumulus model found that acrosome-reacted sperm could penetrate the cumulus in the capillary instead of trapping in the cumulus mass (Rijsdijk and Franken, 2007). It has also been observed that the dispersal of acrosome content persists at least for $15 \mathrm{~min}$ after the initiation of $A R$ in vitro, indicating that the status of acrosome is neither OFF (intact) nor ON (acted), but includes a series of intermediate states (Hardy et al., 1991; Kim et al., 2001; Kim and Gerton, 2003; Sutovsky et al., 2010). Moreover, this model fails to explain why there exists such a large percentage of false or spontaneous acrosome-reacted sperm in almost all species examined so far. If the acrosome enzymes, released as a consequence of the acrosome reaction, are not necessary for sperm to penetrate the cumulus, we should expect to see much more sperm with intact acrosomes, both within the cumulus and on the ZP. Apparently this is not the case when the eggs are surrounded by cumulus, and therefore, the present AR model is obviously inadequate for explaining all the events of acrosome reaction.

\section{CROSS-TALK BETWEEN SPERM AND CUMULUS CELLS}

Controversy still exists regarding the role of the cumulus during fertilization, especially during sperm penetration through the cumulus mass. The role of the cumulus in sperm-egg interaction remains unclear. It should be noted that most in vitro experiments have been focusing on the zona layer with the cumulus cells removed, which might have neglected or overlooked the importance of the cumulus in fertilization. Growing evidence has emerged unfolding the cross-talk between sperm and the cumulus cell and matrix, which requires the re-evaluation of the role of the cumulus in sperm penetration and fertilization.

\section{Facilitated AR in the presence of cumulus layer}

Although the notion that AR occurs in the cumulus layer has been denied for decades, the interaction between sperm and the cumulus mass has been observed constantly. Several lines of evidence have emerged showing that the cumulus could increase sperm AR or even directly induce AR in vitro. In the presence of human cumulus oophorus, a significant enhancement of human sperm AR was observed after $5 \mathrm{~h}$ preincubation with the capacitation medium in vitro (Tesarik, 1985). A study examining the effect of cumulus matrix and cumulus cells on sperm penetration revealed that, compared to cell-depleted matrix, more sperm could penetrate the intact cumulus complex, with a higher percentage of normal morphology, AR and motility (Hong et al., 2009). Fragments of preovulatory human cumulus were washed 3 times and incubated for $24 \mathrm{~h}$, and the spent media and washes were assayed for their ability to initiate human sperm AR in vitro. AR activity was present in the first two washes but not the third wash; however, AR activity was recovered after 3 washes and incubation for $24 \mathrm{~h}$. The percentage of acrosome reacting human sperm was significantly increased if human sperm were incubated with the cumulus mass (Carrell et al., 1993). These findings suggest that interaction of sperm with the cumulus mass could result in $A R$, or that the cumulus mass may facilitate sperm to undergo AR.

\section{Chemoattractants released from cumulus cells}

Another communication between sperm and the cumulus oophorus appears to be the chemotaxis, an important mechanism that enables high forward motility of sperms in the ampulla of fallopian tube, the egg storage site after ovulation, and guides them to the ovulated oocyte. Although the attractants and the molecular mechanism of sperm chemotaxis in mammals are not yet known, sperm chemotaxis to follicular fluid, the mature oocyte and the surrounding cumulus oophorus have been reported by a number of studies (Jeon et al., 2001; Wang et al., 2001; Sun et al., 2005; Gil et al., 2008; Guidobaldi et al., 2008; Oren-Benaroya et al., 2008). It has been postulated that the chemoattractants, including heparin, progesterone, atrial natriuretic peptide (ANP), adrenaline, oxytocin, calcitonin and acetylcholine, might also be originated from the cumulus oophorus (Sliwa, 1993; Sliwa, 1994; Anderson et al., 1995; Sliwa, 1995; VillanuevaDíaz et al., 1995).

\section{Involvement of a sperm-released factor in induction of AR by progesterone of cumulus cells}

Among the potential chemoattractants, it is interesting to note that progesterone is also a potent inducer of $A R$ in vitro. In many species, progesterone is present in the preovulatory follicular fluid and released to the oviduct during ovulation, which might be one of the sources of local progesterone at the site of fertilization (Osman et al., 1989; Thomas and Meizel, 1989). The cumulus cells have also been reported to secret progesterone after ovulation, which is thought to be involved in both sperm chemotaxis to the cumulus oophorus and cumulus-induced acrosome reaction (Witte and SchäferSomi, 2007; Guidobaldi et al., 2008). Therefore progesterone has been suggested to be the trigger of $A R$ in the cumulus layer in vivo. Unfortunately, this process still remains elusive since it is not clear how spermatozoa interact with cumulus cells and trigger the production/release of progesterone, 


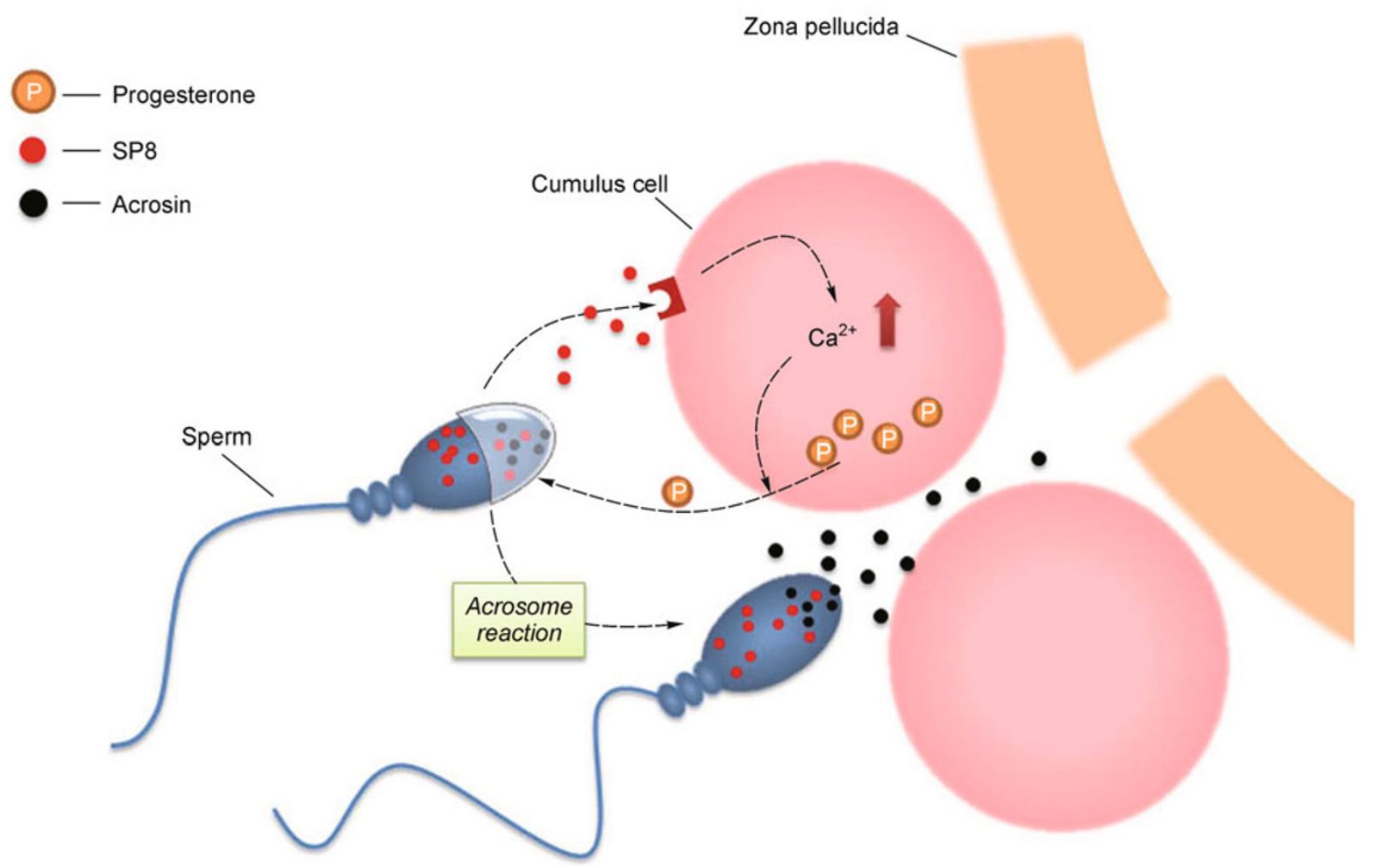

Figure 1. Schematic representation of sperm releasing NYP-SP8 (SP8) and interacting with cumulus cells. As a result of SP8 binding to cumulus cell surface, intracellular calcium of the cumulus cell increases and leads to progesterone release, which subsequently triggers AR to facilitate sperm penetration of the cummulus.

although there have been reports that calcium mobilization is responsible for progesterone production (Jezová et al., 2001; O'Donnell et al., 2004).

Our recent discovery of a sperm factor, which can be released upon sperm-cumulus interaction and induce calcium-dependent progesterone release (Yin et al., 2009), has shed new light into our understanding of AR process during sperm penetration of the cumulus. NYD-SP8 is a glycosylphosphatidylinositol (GPI)-anchored protein localized to the posterior head of human and mouse sperm. When sperm were treated with phosphatidylinositol specific phospholipase C (PI-PLC), which specifically cleaves GPIanchored protein from the membrane, NYD-SP8 could be detected in the supernatant. Moreover, the cumulus cells, which do not express endogenous NYD-SP8, showed a positive NYD-SP8 fluorescent immunostaining after incubation with sperm for six hours, suggesting that NYD-SP8 can be cleaved from sperm membrane upon sperm-cumulus interaction and bind to the cumulus cells. Recombinant NYDSP8 protein significantly increased intracellular calcium in cumulus cells, and induced progesterone production of cumulus cells. This induction could be inhibited by NYDSP8 antibody or calcium chelator BAPTA-AM, suggesting that binding of NYD-SP8 to cumulus cells may lead to production of progesterone in a calcium dependent manner. Moreover, sperm incubation with cumulus cells resulted in an increase of acrosome reaction, and this increase could also be inhibited by NYD-SP8 antibody but reversed by addition of progesterone. These results provide clear evidence of crosstalk between sperms and cumulus mass and cumulusinduced acrosome reaction via a sperm membrane protein, NYD-SP8. It is very likely that NYD-SP8 is cleaved from sperm head upon its interaction with the cumulus and binds to the cumulus cells, which results in calcium mobilization and progesterone production in cumulus cells. The progesterone secreted from cumulus cells subsequently initiates sperm AR within the cumulus layer. The findings of our study have thus provided, for the first time, the possible sequence of events leading to $A R$ in the cumulus (Fig. 1) (Yin et al., 2009).

\section{POTENTIAL ROLE OF THE CUMULUS-INDUCED AR IN FERTILIZATION IN VIVO}

When all the above evidences are pooled together, it is clear that the ZP may not be the only site of AR and that the cumulus mass may also be a physiological site of AR. We thus propose that some sperm initiate their AR when contacting with the cumulus cells, while some of them remain acrosome intact until they reach the ZP and bind to ZP3. This modified model of AR suggests that the cumulus layer is a 
tighter barrier than previously thought, and it requires sperm to undergo $A R$ in order to penetrate through, as they do penetrating ZP. This new AR model reconciles with the above two models and provides a reasonable explanation for the large number of acrosome-reacting or reacted sperm in the cumulus mass observed in many species, which have been previously thought to be "falsely" acrosome-reacted. However, the proposal of AR occurring in the cumulus has encountered a difficulty since acrosome-reacted sperm could not penetrate ZP and enter the egg. Traditionally, people regard spermatozoa as a population but not a sum of millions of individuals, and it is assumed that all sperm initiate their AR at the same point and the same site. However, as individuals, some sperm may initiate their AR in the cumulus and even die before they reach the zona. False AR was proposed to provide a possible explanation for the observed acrosomereacting or reacted sperms located within the cumulus mass. However, it is hard to answer why such a large percentage of sperm go through a wrong reaction. Therefore, a more reasonable explanation might be that the AR initiated at the site of the cumulus is for meaningful purposes that we are not entirely sure at the moment. Obviously, these acrosome reacted sperm may disperse the cumulus mass for other sperm population and it is the sacrifice of the acrosomereacted sperm at the site of cumulus that paves the way for other sperm to follow through without undergoing AR until they reach ZP. This perhaps explains why millions of sperm are required for successful fertilization. AR in the cumulus may serve other purposes, which are still beyond our understanding. For example, the cumulus-induced AR may affect and modify the microenvironment of the cumulus mass, which might have an impact on the other sperm and facilitate them to fertilize the egg.

\section{CONCLUDING REMARK}

AR is an important step that sperm must undergo for successful fertilization, but the exact site of $A R$ in vivo remains controversial. The accepted model that $A R$ occurs at the surface of ZP only upon acrosome-intact sperm binding to ZP3 cannot explain the large number of acrosome-reacted sperm found in the cumulus mass in many species and is challenged by the recent finding of a sperm-released factor, NYD-SP8, that initiates AR upon sperm interaction with cumulus cells. We propose that the cumulus oophorus, in addition to the ZP, is a site for AR initiation. This proposal has suggested the involvement of a sperm protein in the initiation of $A R$ and a possible new role of the cumulus layer in fertilization. Further studies investigating the cross-talk between sperm and the cumulus layer are necessary to confirm this new model and unraveil the mystery of AR in vivo.

\section{ACKNOWLEDGEMENTS}

The authors wish to thank Dr. Yechuan Ruan for the art work. The described work conducted in the authors' lab was supported in parts by the National 973 Project (Nos. 2006CB504002 and 2006CB944002) and the Focused Investment Scheme on Major Areas of The Chinese University of Hong Kong.

\section{REFERENCES}

Anderson, R.A. Jr, Feathergill, K.A., Rawlins, R.G., Mack, S.R., and Zaneveld, L.J. (1995). Atrial natriuretic peptide: a chemoattractant of human spermatozoa by a guanylate cyclase-dependent pathway. Mol Reprod Dev 40, 371-378.

Baba, D., Kashiwabara, S., Honda, A., Yamagata, K., Wu, Q., Ikawa, M., Okabe, M., and Baba, T. (2002). Mouse sperm lacking cell surface hyaluronidase $\mathrm{PH}-20$ can pass through the layer of cumulus cells and fertilize the egg. J Biol Chem 277, 30310-30314.

Bedford, J.M. (1968). Ultrastructural changes in the sperm head during fertilization in the rabbit. Am J Anat 123, 329-358.

Bedford, J.M. (1972). An electron microscopic study of sperm penetration into the rabbit egg after natural mating. Am $\mathrm{J}$ Anat 133, 213-254.

Bleil, J.D., and Wassarman, P.M. (1980). Mammalian sperm-egg interaction: identification of a glycoprotein in mouse egg zonae pellucidae possessing receptor activity for sperm. Cell 20, 873-882.

Bleil, J.D., and Wassarman, P.M. (1983). Sperm-egg interactions in the mouse: sequence of events and induction of the acrosome reaction by a zona pellucida glycoprotein. Dev Biol 95, 317-324.

Breitbart, H., Rotman, T., Rubinstein, S., and Etkovitz, N. (2010). Role and regulation of $\mathrm{PI} 3 \mathrm{~K}$ in sperm capacitation and the acrosome reaction. Mol Cell Endocrinol 314, 234-238.

Brucker, C., and Lipford, G.B. (1995). The human sperm acrosome reaction: physiology and regulatory mechanisms. An update. Hum Reprod Update 1, 51-62.

Carrell, D.T., Middleton, R.G., Peterson, C.M., Jones, K.P., and Urry, R.L. (1993). Role of the cumulus in the selection of morphologically normal sperm and induction of the acrosome reaction during human in vitro fertilization. Arch Androl 31, 133-137.

Cross, N.L., Morales, P., Overstreet, J.W., and Hanson, F.W. (1988). Induction of acrosome reactions by the human zona pellucida. Biol Reprod 38, 235-244.

Crozet, N. (1984b). Ultrastructural aspects of in vivo fertilization in the cow. Gamete Res 10, 241-251.

Crozet, N., and Dumont, M. (1984a). The site of the acrosome reaction during in vivo penetration of the sheep oocyte. Gamete Res 10, 97-105.

Cummins, J.M., and Yanagimachi, R. (1982). Sperm-egg ratios and the site of the acrosome reaction during in vivo fertilization in the hamster. Gamete Res 5, 239-256.

Fukami, K., Nakao, K., Inoue, T., Kataoka, Y., Kurokawa, M., Fissore, R.A., Nakamura, K., Katsuki, M., Mikoshiba, K., Yoshida, N., et al. (2001). Requirement of phospholipase Cdelta4 for the zona pellucida-induced acrosome reaction. Science 292, 920-923.

Gil, P.I., Guidobaldi, H.A., Teves, M.E., Uñates, D.R., Sanchez, R., and Giojalas, L.C. (2008). Chemotactic response of frozen-thawed bovine spermatozoa towards follicular fluid. Anim Reprod Sci 108, 236-246.

Guidobaldi, H.A., Teves, M.E., Uñates, D.R., Anastasía, A., and 
Giojalas, L.C. (2008). Progesterone from the cumulus cells is the sperm chemoattractant secreted by the rabbit oocyte cumulus complex. PLoS One 3, e3040.

Gupta, S.K., Bansal, P., Ganguly, A., Bhandari, B., and Chakrabarti, K. (2009). Human zona pellucida glycoproteins: functional relevance during fertilization. J Reprod Immunol 83, 50-55.

Gwatkin, R.B.L., Carter, H.W., and Patterson, H. (1976). Association of mammalian sperm with the cumulus cells and the zona pellucida studied by scanning electron microscopy. In: Scanning Electron Microscopy, Proceedings of Workshopon SEM in Reproductive Biology, Part 2. Johari, O. ed. Chicago: IIT Res Inst. 379-384.

Hardy, D.M., Oda, M.N., Friend, D.S., and Huang, T.T. Jr. (1991). A mechanism for differential release of acrosomal enzymes during the acrosome reaction. Biochem J 275, 759-766.

Hasuwa, H., Muro, Y., Ikawa, M., Kato, N., Tsujimoto, Y., and Okabe, M. (2010). Transgenic mouse sperm that have green acrosome and red mitochondria allow visualization of sperm and their acrosome reaction in vivo. Exp Anim 59, 105-107.

Hong, S.J., Chiu, P.C., Lee, K.F., Tse, J.Y., Ho, P.C., and Yeung, W.S. (2009). Cumulus cells and their extracellular matrix affect the quality of the spermatozoa penetrating the cumulus mass. Fertil Steril 92, 971-978.

Ikawa, M., Inoue, N., Benham, A.M., and Okabe, M. (2010). Fertilization: a sperm's journey to and interaction with the oocyte. J Clin Invest 120, 984-994.

Jeon, B.G., Moon, J.S., Kim, K.C., Lee, H.J., Choe, S.Y., and Rho, G. J. (2001). Follicular fluid enhances sperm attraction and its motility in human. J Assist Reprod Genet 18, 407-412.

Jezová, M., Scsuková, S., Nagyová, E., Vranová, J., Procházka, R., and Kolena, J. (2001). Effect of intraovarian factors on porcine follicular cells: cumulus expansion, granulosa and cumulus cell progesterone production. Anim Reprod Sci 65, 115-126.

Kim, E., Baba, D., Kimura, M., Yamashita, M., Kashiwabara, S., and Baba, T. (2005). Identification of a hyaluronidase, Hyal5, involved in penetration of mouse sperm through cumulus mass. Proc Natl Acad Sci U S A 102, 18028-18033.

Kim, K.S., Foster, J.A., and Gerton, G.L. (2001). Differential release of guinea pig sperm acrosomal components during exocytosis. Biol Reprod 64, 148-156.

Kim, K.S., and Gerton, G.L. (2003). Differential release of soluble and matrix components: evidence for intermediate states of secretion during spontaneous acrosomal exocytosis in mouse sperm. Dev Biol 264, 141-152.

Kimura, M., Kim, E., Kang, W., Yamashita, M., Saigo, M., Yamazaki, T., Nakanishi, T., Kashiwabara, S., and Baba, T. (2009). Functional roles of mouse sperm hyaluronidases, HYAL5 and SPAM1, in fertilization. Biol Reprod 81, 939-947.

Lee, M.A., Check, J.H., and Kopf, G.S. (1992). A guanine nucleotidebinding regulatory protein in human sperm mediates acrosomal exocytosis induced by the human zona pellucida. Mol Reprod Dev 31, 78-86.

Lin, Y., Mahan, K., Lathrop, W.F., Myles, D.G., and Primakoff, P. (1994). A hyaluronidase activity of the sperm plasma membrane protein $\mathrm{PH}-20$ enables sperm to penetrate the cumulus cell layer surrounding the egg. J Cell Biol 125, 1157-1163.

Miller, D.J., Macek, M.B., and Shur, B.D. (1992). Complementarity between sperm surface beta-1,4-galactosyltransferase and eggcoat ZP3 mediates sperm-egg binding. Nature 357, 589-593.
Nakanishi, T., Ikawa, M., Yamada, S., Parvinen, M., Baba, T., Nishimune, Y., and Okabe, M. (1999). Real-time observation of acrosomal dispersal from mouse sperm using GFP as a marker protein. FEBS Lett 449, 277-283.

Nakanishi, T., Ikawa, M., Yamada, S., Toshimori, K., and Okabe, M. (2001). Alkalinization of acrosome measured by GFP as a $\mathrm{pH}$ indicator and its relation to sperm capacitation. Dev Biol 237, 222-231.

O'Donnell, J.B. Jr, Hill, J.L., and Gross, D.J. (2004). Epidermal growth factor activates cytosolic $\left[\mathrm{Ca}^{2+}\right]$ elevations and subsequent membrane permeabilization in mouse cumulus-oocyte complexes. Reproduction 127, 207-220.

Oren-Benaroya, R., Orvieto, R., Gakamsky, A., Pinchasov, M., and Eisenbach, M. (2008). The sperm chemoattractant secreted from human cumulus cells is progesterone. Hum Reprod 23, 2339-2345.

Osman, R.A., Andria, M.L., Jones, A.D., and Meizel, S. (1989). Steroid induced exocytosis: the human sperm acrosome reaction. Biochem Biophys Res Commun 160, 828-833.

Pereda, J., and Coppo, M. (1985). An electron microscopic study of sperm penetration into the human egg investments. Anat Embryol (Berl) 173, 247-252.

Rijsdijk, M., and Franken, D.R. (2007). Use of the capillary-cumulus oophorus model for evaluating the selection of spermatozoa. Fertil Steril 88, 1595-1602.

Sliwa, L. (1993). Heparin as a chemoattractant for mouse spermatozoa. Arch Androl 31, 149-152.

Sliwa, L. (1994). Chemotactic effect of hormones in mouse spermatozoa. Arch Androl 32, 83-88.

Sliwa, L. (1995). Chemotaction of mouse spermatozoa induced by certain hormones. Arch Androl 35, 105-110.

Sun, F., Bahat, A., Gakamsky, A., Girsh, E., Katz, N., Giojalas, L.C., Tur-Kaspa, I., and Eisenbach, M. (2005). Human sperm chemotaxis: both the oocyte and its surrounding cumulus cells secrete sperm chemoattractants. Hum Reprod 20, 761-767.

Sutovsky, P.Sperm capacitation, the acrosome reaction and fertilization. (2010). In: Reproductive Endocrinology and Infertility: Integrating Modern Clinical and Laboratory Practice. Carrell, D. T. and Peterson, C.M., eds. New York: Springer Science Business Media. 389-421.

Talbot, P. (1985). Sperm penetration through oocyte investments in mammals. Am J Anat 174, 331-346.

Tanii, I., Aradate, T., Matsuda, K., Komiya, A., and Fuse, H. (2011). PACAP-mediated sperm-cumulus cell interaction promotes fertilization. Reproduction 141, 163-171.

Tesarík, J. (1985). Comparison of acrosome reaction-inducing activities of human cumulus oophorus, follicular fluid and ionophore A23187 in human sperm populations of proven fertilizing ability in vitro. J Reprod Fertil 74, 383-388.

Thomas, P., and Meizel, S. (1989). Phosphatidylinositol 4,5-bisphosphate hydrolysis in human sperm stimulated with follicular fluid or progesterone is dependent upon $\mathrm{Ca}^{2+}$ influx. Biochem $\mathrm{J} 264$, 539-546.

Tulsiani, D.R.P., Abou-Haila, A., Loeser, C.R., and Pereira, B.M. (1998). The biological and functional significance of the sperm acrosome and acrosomal enzymes in mammalian fertilization. Exp Cell Res 240, 151-164.

van Duin, M., Polman, J.E.M., De Breet, I.T.M., van Ginneken, K., 
Bunschoten, H., Grootenhuis, A., Brindle, J., and Aitken, R.J. (1994). Recombinant human zona pellucida protein ZP3 produced by chinese hamster ovary cells induces the human sperm acrosome reaction and promotes sperm-egg fusion. Biol Reprod 51, 607-617.

Villanueva-Díaz, C., Arias-Martínez, J., Bermejo-Martínez, L., and Vadillo-Ortega, F. (1995). Progesterone induces human sperm chemotaxis. Fertil Steril 64, 1183-1188.

Wang, Y., Storeng, R., Dale, P.O., Abyholm, T., and Tanbo, T. (2001). Effects of follicular fluid and steroid hormones on chemotaxis and motility of human spermatozoa in vitro. Gynecol Endocrinol 15, 286-292.

Witte, T.S., and Schäfer-Somi, S. (2007). Involvement of cholesterol, calcium and progesterone in the induction of capacitation and acrosome reaction of mammalian spermatozoa. Anim Reprod Sci 102, 181-193.

Yanagimachi, R. (1988). Mammalian fertilization. In: The Physiology of Reproduction, 1st edi. E.Knobil and J.D.Neil, eds. New York: Raven Press. 135-185.

Yanagimachi, R. (1994). Mammalian fertilization. In: The Physiology of Reproduction, 2nd edi. E.Knobil and J.D.Neil, eds. New York: Raven Press. 189-317.

Yin, L., Chung, C.M., Huo, R., Liu, H., Zhou, C., Xu, W., Zhu, H., Zhang, J., Shi, Q., Wong, H.Y., et al. (2009). A sperm GPIanchored protein elicits sperm-cumulus cross-talk leading to the acrosome reaction. Cell Mol Life Sci 66, 900-908. 